\title{
Étude thermodynamique de 4 cristaux liquides contenant des substituants dioxyethylene par analyse enthalpique differentielle et chromatographie en phase gazeuse
}

\author{
M.H. Guermouche ${ }^{1}$, S. Guermouche ${ }^{1}$, F. Ammar-Khodja ${ }^{1}$, J.P. Bayle ${ }^{2}$ \\ et M. Rogalski ${ }^{3}$ \\ ${ }^{1}$ Laboratoire de Chromatographie, Faculté de Chimie, USTHB, BP. 32 El-Alia, \\ Alger, Algérie \\ ${ }^{2}$ Laboratoire de Chimie Structurale Organique, ICMO, Bât. 410, 91405 Orsay \\ Cedex, France \\ ${ }^{3}$ Laboratoire de Thermodynamique et d'Analyse Chimique, Université de \\ Metz, France
}

\begin{abstract}
Summary. A thermodynamic study of 4 liquid crystals with DOE substituents using gas chromatography are carried out. The 4 compounds have the same mesogenic part; they differ by the partial or total substitution of the terminal chain heptyloxy with 2-(2-methoxyethoxy)ethoxy (DOE). The thermal properties of the 4 compounds are determined by differential scaning calorimetry. The knowledge of the gas chromatographic specific retention volume of a serial geometric isomers obtained by using the 4 liquid crystals as stationary phases allow to calculate their thermodynamic data : activity coeffients, enthalpies and entropies of solution. In the other hand, the support influence on the thermal properties are investigated. The results founds show that this parameter is not negligible but disappear when the coating increases. For silica, the transitions (melting, clarification) are apparent only when the coating is higher than $50 \%$. With Chromosorb P, $5 \%$ of coating are sufficient to observe the transitions.
\end{abstract}

\section{INTRODUCTION}

Les cristaux liquides ont été utilisés intensivement comme phases stationnaires en chromatographie. Très peu de travaux théoriques sont relatifs à la compréhension de la rétention des solutés. De plus, l'influence du support chromatographique sur l'orientation des molécules de cristaux liquides a été certes évoquée mais pas étudiée. Dans ce travail, en utilisant des cristaux liquides ayant une grande similitude de structure $[1,2]$, nous nous proposons de déterminer les grandeurs thermodynamiques de solution et d'excès de différents solutés et d'étudier l'influence des supports sur les températures de transition

\subsection{Partie théorique}

Le volume de rétention spécifique $V g$ d'un soluté est déterminé par [3]:

$$
\ln (\mathrm{Vg})=-\Delta H / R T+\Delta S / R-\ln \left(M_{1} / 273.16 R\right)=-\Delta G / R T-\ln \left(M_{1} / 273.16 R\right)
$$

$\mathrm{M}_{1}$ est la masse molaire de la phase stationnaire. Le sens physique de $\Delta H, \Delta S$ et $\Delta G$ dépend de l'état physique de la phase stationnaire. Dans l'état solide, $A H, A S$ et $\Delta G$ correspondent respectivement à l'enthalpie molaire, l'entropie et l'enthalpie libre molaire d'adsorption. Pour une phase nématique ou liquide, le soluté est dissous dans la phase stationnaire et $A H, \Delta S$ et $\Delta G$ représentent l'enthalpie molaire, l'entropie molaire et l'enthalpie libre molaire de dissolution

Le coefficient d'activité à dilution infinie d'un soluté dans une phase nématique ou liquide est donné par

$$
\ln \gamma_{2}^{\infty}=\ln \left[273 \mathrm{R} /\left(\mathrm{Vg} \cdot \mathrm{p}_{2}^{\mathrm{s}} \cdot \mathrm{M}_{1}\right)\right]-B_{22} \mathrm{p}_{2}^{\mathrm{s}} / \mathrm{RT}
$$


$\mathrm{p}_{2}$ est la pression de vapeur du soluté à la température $\mathrm{T}, B_{22}$ est le second coefficient du viriel du soluté. Les grandeurs d'excès peuvent alors être déterminées par :

$$
G_{2}^{e, \infty}=\mathrm{RT} \cdot \ln \left(\gamma_{2}^{\infty}\right)=H_{2}^{e, \infty}-T S_{2}^{e, \infty}
$$

\subsection{Partie expérimentale}

Le chromatographe utilisé est un HP 5730A équipé d'un FID. Les colonnes en acier utilisées (0.91m. ' $3 \mathrm{~mm}$ I.D) sont remplies avec la phase stationnaire (Chromosorb W HP (0.125-0.14 $\mathrm{mm})+10 \%$ de cristal liquide. Le gaz vecteur $(\mathrm{He})$ a un débit de $30 \mathrm{~mL} \cdot \mathrm{min}^{-1}$. Les mesures DSC ont été réalisées sur un appareil TA instrument .

La figure 1 donne la structure des cristaux liquides étudiés<smiles>[R]Oc1ccc(N=Nc2ccc(OC(=O)c3ccc(O[R])cc3)cc2)cc1</smiles>

$\mathrm{C}_{7}-\mathrm{C}_{7}$

DOE $-\mathrm{C}_{7}$

$\mathrm{C}_{7}=\mathrm{DOE}$

DOE -DOE

$$
\begin{gathered}
\mathrm{R}_{1} \\
\mathrm{CH}_{3}-\left(\mathrm{CH}_{2}\right)_{6} \\
\left(\mathrm{CH}_{2}\right)_{2} \mathrm{O}-\left(\mathrm{CH}_{2}\right)_{2} \mathrm{O}-\mathrm{CH}_{3} \\
\mathrm{CH}_{3}-\left(\mathrm{CH}_{2}\right)_{6} \\
\left(\mathrm{CH}_{2}\right)_{2} \mathrm{O}-\left(\mathrm{CH}_{2}\right)_{2}-\mathrm{O}-\mathrm{CH}_{3}
\end{gathered}
$$

\author{
$\mathbf{R}_{\mathbf{2}}$ \\ $\mathrm{CH}_{3}-\left(\mathrm{CH}_{2}\right)_{6}$ \\ $\mathrm{CH}_{3}-\left(\mathrm{CH}_{2}\right)_{6}$ \\ $\left(\mathrm{CH}_{2}\right)_{2} \mathrm{O}-\left(\mathrm{CH}_{2}\right)_{2} \mathrm{O}-\mathrm{CH}_{3}$ \\ $\left(\mathrm{CH}_{2}\right)_{2} \mathrm{O}-\left(\mathrm{CH}_{2}\right)_{2}-\mathrm{O}-\mathrm{CH}_{3}$
}

Figure 1. Structure des cristaux liquides étudiés

\subsection{Résultats et discussion}

\subsubsection{Grandeurs de solution et d'excès}

Les soluté considérés ont été injectés à différentes températures, les temps de rétention ont permis de déterminer leur volume de rétention spécifiques.

La figure 2 montre un exemple de variation de $\ln \Delta G$ en fonction de la température mettant en évidence les 3 états physiques des composés étudiés et les transitions correspondantes; la transition solide nématique ou fusion et la transition nématique liquide ou clarification..

L'équation (1) permet de relier la pente et ordonnée à l'origine des droites apparaissant sur la figure (1) aux grandeurs thermodynamiques d'adsorption dans le cas de la phase solide et aux grandeurs thermodynamiques de solution dans les états nématique ou liquide 


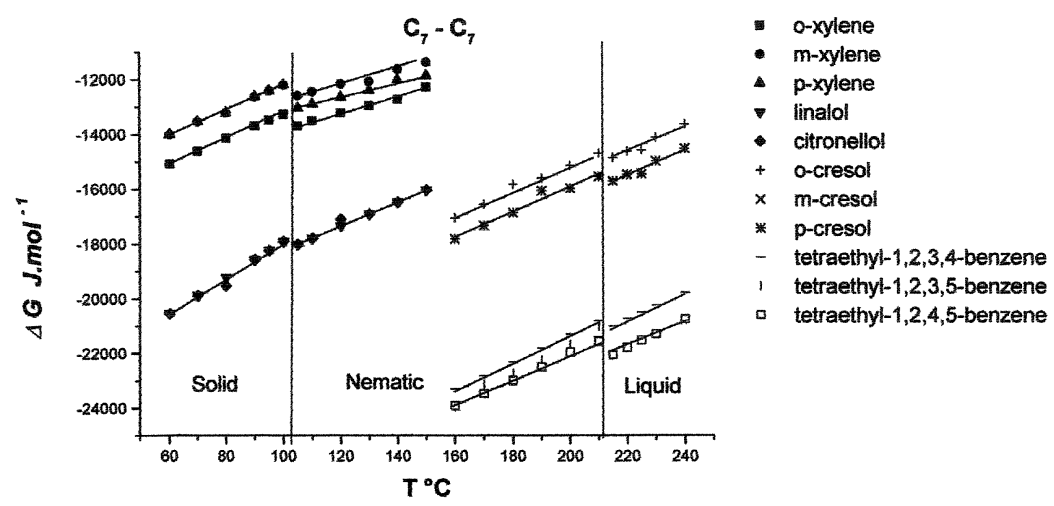

Figure 2. variation de $\Delta G$ en fonction de la température.

La figure 3 montre un exemple de la variation de $G_{2}{ }^{e, \infty}$ en fonction de la température dans le domaine nématique ou liquide. Les grandeurs d'excès $H_{2}^{e, \infty}$ et $S_{2}^{e, \infty}$ sont obtenues à partir des pentes et ordonnées à l'origine de ces droites.

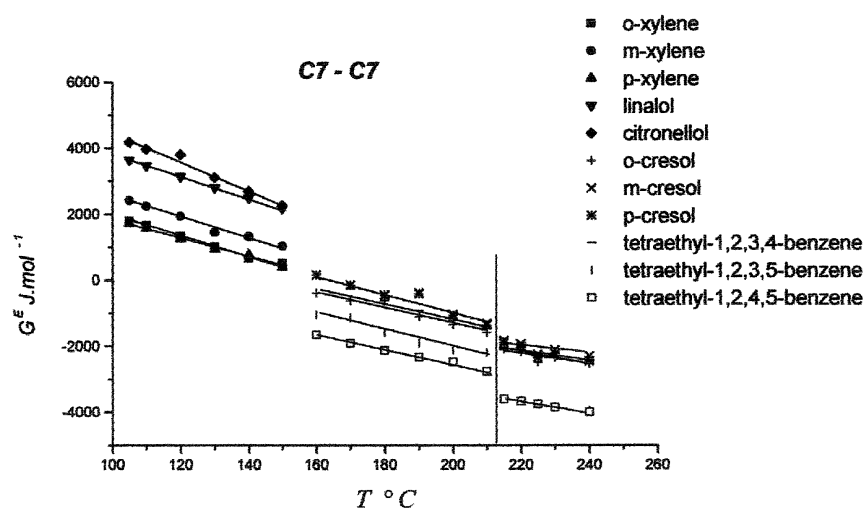

Figure 3. variation de $G_{2}^{e, \infty}$ en fonction de la température; cas du $C_{7}-C_{7}$.

\subsubsection{Etude par DSC de l'influence de la nature du support sur les transitions}

A titre d'exemple, pour les cristaux liquides $C_{7}-C_{7}$ et $D O E-C_{7}$, la figure 5 montre les thermogrammes du produit pur, du recouvrement du Chromosorb WHP et de la silice avec $10 \%$ de cristal liquide. De plus, dans le cas du DOE-C $C_{7}$, le thermogramme supplémentaire est relatif à la silice recouverte de $50 \%$ de cristal liquide. 
Dans le cas des supports classiques du type Chromosorb WHP, les transitions solidenématique et nématique liquide ne sont pas perturbées avec un recouvrement de $10 \%$ par le cristal liquide. Les transitions solide-solide n'apparaissent plus (voir $\mathrm{C}_{7}-\mathrm{C}_{7}$ par exemple)

Dans le cas des supports à forte surface spécifique comme la silice $\left(300 \mathrm{~m}^{2} \cdot \mathrm{g}^{-1}\right)$, les transitions solide-nématique et nématique liquide n'apparaissent plus avec un recouvrement de $10 \%$. Elles réapparaissent et sont parfois perturbées avec un recouvrement de $50 \%$.

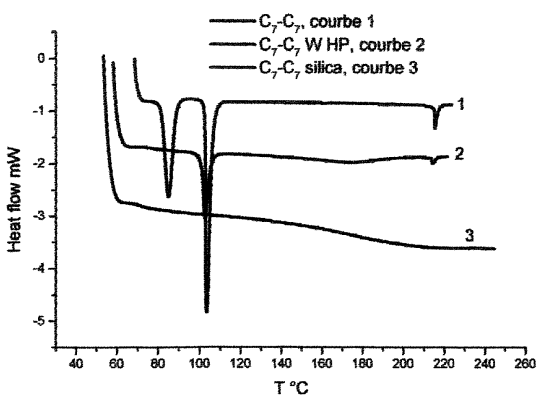

a)

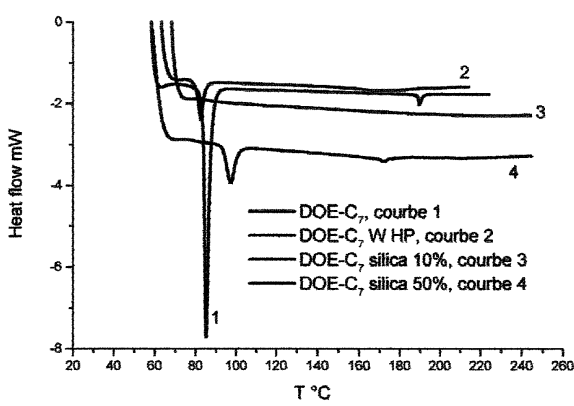

b)

Figure 5. thermogrammes du produit pur, du recouvrement du Chromosorb WHP et de la silice par le cristal liquide. $\mathrm{C}_{7}-\mathrm{C}_{7}$ (a) ou DOE- $\mathrm{C}_{7}$ (b)

\subsection{Conclusion}

Les résultats indiquent que les relations classiques de rétention permettent d'avoir les grandeurs thermodynamiques directement liées aux propriétés séparatives des phases étudiées. Les courbes DSC ont permis de préciser l'influence du support sur les transitions. Avec un support classique de type WHP, la fusion et la clarification ne sont pas perturbées avec un recouvrement de $10 \%$.

\section{Références}

1.P.Judeinstein , P.Berdagué , J.P.Bayle, Ewa Rogalska, Marek Rogalski, D.Petit Jean, M.H.Guermouche, J.Chromatogr.A, 859, 59 (1999)

2.F.Ammar-Khodja,S.Guermouche, M.H. Guermouche, P.Berdague, J.P.Bayle., Chromatographia, 50,338 (1999).

3.L. C. Chow, D. E. Martire, J. Phys. Chem., 75, 2005 (1971) 